\title{
Modified Artificial Potential Field Method for Online Path Planning Applications
}

\author{
Farid Bounini, Denis Gingras \\ LIV - Université de Sherbrooke \\ Sherbrooke, Canada \\ farid.bounini@usherbrooke.ca \\ denis.gingras@usherbrooke.ca
}

\author{
Herve Pollart, \\ OPAL-RT Technologies Inc, \\ Montreal, Canada \\ herve.pollart@opal-rt.com
}

\author{
Dominique Gruyer, \\ LIVIC, \\ IFSTTAR, France \\ dominique.gruyer@ifsttar.fr
}

\begin{abstract}
This paper presents a modified potential field method for robot navigation. The approach overcomes the wellknown artificial potential field (APF) method issue, which is due to local minima that induce the standard APF method to trap in. Thus, the standard APF method is no longer useful in such case. The advantage of the new proposed method, as opposed to those that resort to the global optimization methods, is the low computing time that lines up with the standard A-Star $\left(A^{*}\right)$ method. The strategy consists of looking for a practical path in the potential field-according to the potential gradient descent algorithm (PGDA) — and adding a repulsive potential to the current state, in case of blocking configuration, a local minimum. When the PGDA reaches the global minimum, a new potential field will be constructed with only one minimum that matches the final destination of the robot, the global minimum. Finally, to determine the achievable trajectory, a second iteration is performed by the PGDA.
\end{abstract}

Keywords-Path Planning; Artificial Potential Field Method; Potential Gradient Descent Algorithm; A* algorithm.

\section{INTRODUCTION}

One of the most important branches of artificial intelligence is trajectory planning, which is applied to robotic navigation and self-driving vehicles. It consists of finding a set of sequences that allows a robot to travel from an initial to a global state [1]. It could also be defined as moving furniture in a house without colliding with or touching walls and the other objects [1]. In the early work, the robots' operational environment is considered to be deterministic or inert, especially deployed in and for industrial environment [2]. Gradually, developments in technology enabled the new robotic generations to become partially autonomous. Therefore, the robot must be suitably equipped with the perception, localization, data fusion, decision making, and control abilities. If the first generation of robots were equipped with simple integrated circuits for fixed-control programs-relying on basic intelligence to repeat a series of actions in a static environment - the coming generations will require a significantly more sophisticated artificial intelligence [2]. In fact, these needs are a consequence of the increase in the number of degrees of freedom (DOF) of self-governing robots. The new generation, so-called third generation, must be able to map their trajectories and react instantaneously to their surroundings [1]. Another aspect of artificial intelligence, which characterizes a smart robot, is traveling from its current position to its destination by determining a feasible path autonomously [3], without involving humans. In other words, the robot moves on its own. Most of the decisions are made fully autonomously: meaning that itinerary (the planned route), obstacle detection and avoidance, the robots' dynamic control, and communication with its environment (other robots, infrastructure, etc.) will be made autonomously. As a consequence, the requirements for a self-driving robot result in the development of a new robotics field on a large scale, and in an uncertain environment. Therefore, artificial intelligence is definitely changing the operating mode of the existing robots and will certainly continue towards making them completely autonomous. Furthermore, intelligent robots are influencing manufacturers and their vision of their interaction with humans. This is mainly due to the improvement in semiconductors technologies that provide computers with high computing abilities, enabling complex operations, such as data processing, control, planning, etc., and with sufficient memory to store all incoming and outgoing data to and from the robots' environment (which is needed for decision making algorithms and robot control).

This paper describes a new approach for robot real-time navigation in static and highly dynamic environments. The strategy is based on a modified potential field method (MPFM). The proposed MPFM eliminates local minima (which are due to the robots environment configuration), and find a practical trajectory for robot path planning. The paper incorporates a total of six parts: The first part is a short introduction to autonomous robot evolution in static and non-deterministic environment that requires some basic abilities, and artificial intelligence, that are based on the robot-sensing equipment. The second part mainly presents some related work on trajectory planning algorithms based on artificial potential field method, or combined with other path planning methods. The third part describes two classical trajectory planning algorithms: $A^{*}$ and artificial potential field methods, according to literature, that highlights some of their advantages and drawbacks. In the fourth part, the major problem of the potential field method 
is presented and solved by the suggested strategy, which overcomes the standard APF local minima and adapted for online path planning. In the fifth one, some simulation results are presented and discussed by comparing the two methods, where the results of standard $\mathrm{A}^{*}$ trajectory planning are compared with those of the proposed MAPF method. Finally, the last part concludes the paper and mentions future work.

\section{Related WORK}

The perception is often ranked among the three central aspects of an intelligent robot. In this work, we assume that the proprioceptive sensors data fusion is already performed according to specific algorithms. For instance, in [4], You $\mathrm{Li}$ et al. deal with the perception issues frequently encountered in smart systems. The problem was merging data that came from two on-board pinhole cameras and a Light Detection And Ranging (LIDAR). The algorithm estimates the rotation and translation parameters from the LIDAR coordinate system to the stereoscopic coordinate system (the left camera is taken as the main stereo system). The different intrinsic and extrinsic parameters are determined through simulation and experiments on a specific panel (a chessboard panel modeled by a 3D plane), according to the proposed methods in [4-7].

The artificial potential field (APF) method is widely used throughout the literature. It is based on the uptake of the robot to a particle, constrained to move in an APF. The field is a combination of an attractive field, assigned to the global position or to the target, and a set of repulsive fields related to the obstacles around the robot in its environment [8-10]. The robot's trajectory is determined iteratively by a potential gradient descent algorithm. The concept was initially introduced by Khatib [11] to control a manipulator motion, avoid neighboring obstacles, and plan the arm's motion. The artificial potential field (APF) method is a simple and very effective strategy for path planning. It is quite fast for online trajectory planning [12]. Thanks to its simple concept and effectiveness for online applications, the APF method is applied in different planning challenges, for instance: Ferry Rippun et al. [13] applied the potential field method on robotic soccer, which, in their study, was implemented in a multi-cooperative autonomous robots. The proposed approach called Double Target Potential Field (DTPF). The main objective was to increase the effectiveness of the robot movement and achieve two challenging actions: 1-passing the ball to another robot, and 2-kicking the ball into the goal. To solve the path planning problem in the $3 \mathrm{D}$ environment for the rotarywing flying robot, Jianhao Tan et al. resort, in their work [14], to artificial potential field method to smooth the offline predetermined $A^{*}$ path (which is indeed the shortest path but a non-smooth one); it goes from the robot to the target according to the flying robot's environment. The predetermined $\mathrm{A}^{*}$ trajectory is used as a reference in the case of APF method to smooth the flying robot trajectory for online applications. The combination of the two methods-APF and A*-solved the shortcomings of both methods (non-smoothness and local minima issues, respectively). The APF method has been extended to address problems in which the goal is not reachable due to the obstacle proximity [15], and navigation in narrow passages and in unknown semi-structured environments [16]. Other recent work [17] has focused on the modification of the computation of the artificial potential field according to some fuzzy criteria, which are added to the APF path planning method. The strategy was made up according to: 1-the velocity vector of the robot, 2-the modified potential field force function, and 3-their integration of the fuzzy controller, which consists of adjusting the factors of repulsive potential field in real time.

\section{Trajectory PlanNing Methods}

One of the most crucial tasks of autonomous robots is path planning. It based on sensing the robot's environment [1]. The robot shapes its surrounding environment into two or three dimensions and locates itself in it. Then, it relies to trajectory planning algorithms to determine its path and avoid any collision with the obstacles. The path planning methods are multiple, and the most-used approaches are highlighted in [1]: visibility graph, cell decomposition, Dijkstra, etc. This section describes two path planning methods: $A^{*}$ is a well-known robot trajectory planning that will be compared to the proposed modified artificial potential field method to overcome the local minima issue.

$A^{*}$ is one of the most effective trajectory planning methods. It is used to solve robot navigation issues in congested environment and in multiple digital games such as mazes [18]. The fundamental idea is to find the shortest path, and to optimize Dijkstra's criterion [19]. A* uses Euclidean norm to evaluate the distance between the different states. The basic algorithm does not take into account the obstacles between the current state and the final state. The procedures that are used to overcome such issues (like when the robot gets in a dead node) are: 1- To look for other paths when the robot is in a blocking node; 2- To avoid any path leading to blocking nodes. To do so, two path lists are created. The first is the OPENED list, it includes the set of paths to explore, in order to find the optimal path. The second is the CLOSED list leading to dead nodes. Sometimes, this path searching is timeconsuming. To overcome such constraints, optimization criteria are associated with the planning issue. Some others opted for heuristic solutions as suggested in [20].

The derivatives of the APF method can be easily implemented and executed in real time for control and navigation purposes [11-12]. The basic concept of the robot motion, in the potential field, may be interpreted as a moving particle in a field of two electric particles with different signs. Analogically, the positively charged particle is the robot. The global position is negatively charged, and the obstacles are considered as a set of particles with the same charge as the robot. The field potential gradient may be interpreted as forces that 
constrain the positive particle to reach the position of the negative one. The barriers create repulsive forces and push the robot to move away from obstacles [8-10]. At each configuration $Q$, the total APF forms an attractive force that is defined as the negative gradient of the field. It denotes the favorable direction of the robot motion to reach the global position $Q_{g}$ safely. The combination of the attractive and the repulsive forces drives the robot to its destination.

Many forms of the positive potential exist, the most commonly used throughout the literature are parabolic and conical functions. The robot takes advantage of the second function when it is far away from the global state. And it uses the first once it gets very close to the target, e.g. in a circle defined by its center that corresponds to the global position and some specific radius $R_{g}$.

Parabolic functions: The mathematical expression (1) formulates the shape of the parabolic APF.

$U^{a_{p}}(Q)=\frac{1}{2} k^{a}(d(Q))^{2}$

where $k^{a}>0$ is a positive constant, known as gravitational constant, $d(Q)=\left\|Q-Q_{g}\right\|$ is the Euclidean distance between the state $Q$ and the global state $Q_{g}$. $Q_{g}=\left(x_{g}, y_{g}\right)$ and $Q=(x, y)$ in $2 \mathrm{D}$, or $Q_{g}=\left(x_{g}, y_{g}, z_{g}\right)$ and $Q=(x, y, z)$ in $3 \mathrm{D}$.

The function (1) is perpetually positive, and its global minimum equals to zero. The approach assigns the highest potential field to the starting state. It can be considered as a particle of a mass " $m$ " located at a relative height " $h$ " to the global state. The particle holds a potential energy " $E_{p}=m h$ ", which is transformed into kinetic energy as the robot approaches its destination. The APF shape provides the robot the best direction and the shortest path. The potential gradient is defined as a proportional vector of the difference between the global $Q_{g}$ and the current $Q$ states. The further the robot is from its endpoint, the greater is the gradient, and the bigger the attractive forces. The resulting force of this field is the negative gradient of the APF as given in equation (2). The force also decreases as the robot is getting closer to the global state. Once there, the resultant force becomes null, which allows the robot to reach its endpoint and stay at its destination. This process is achieved repeatedly for each novel destination.

$$
f^{a_{p}}(Q)=-\nabla U^{a_{p}}=-k^{a}\left(Q-Q_{g}\right)
$$

Conical function: The conical AFPs form is dissimilar to the parabolic AFPs from. The two functions are equal at the periphery of the unit circle with the target coordinates $Q_{g}$ as its center. The mathematical formulation (3) defines the shape of the conical potential and its negative gradient.

$$
\left\{\begin{array}{l}
U^{a_{c}}(Q)=\frac{1}{2} k^{a} d(Q) \\
f^{a_{c}}(Q)=-\nabla U^{a_{c}}(Q)=-\frac{k^{a}\left(Q-Q_{g}\right)}{d(Q)}
\end{array}\right.
$$

Repulsive potential field: Several forms are quoted in literature. Khatib proposed the function (4) as a repulsive APF $[9,11]$. It associates to every obstacle a repulsive APF and affects each spatial cell according to the equation (4).

$U_{i}^{r}(Q)=\left\{\begin{array}{l}\frac{1}{2} k_{i}^{r}\left(\frac{1}{d_{i}(Q)}-\frac{1}{d_{i}^{o}}\right)^{2} \text { if } d(Q) \leq d_{i}^{o} \\ 0 \quad \text { otherwise }\end{array}\right.$

where $k_{i}^{r}>0$ is a positive constant. $d_{i}{ }^{o}$ is the distance of influence of the $\mathrm{i}^{\text {th }}$ obstacle. $d_{i}(Q)$ is the smallest distance between the current robot position and the $i^{\text {th }}$ obstacle. The potential field is defined as being zero outside the distance of the influence of the objects, positive inside, and infinite above the objects.

$$
d_{i}(Q)=\min _{Q^{\prime} \in Q_{i}^{o}}\left\|Q-Q^{\prime}\right\|
$$

where $Q_{i}^{o}$ is the position coordinates of the $\mathrm{i}^{\text {th }}$ obstacle.

The resulting forces are expressed as follows.

$$
\begin{aligned}
f_{i}^{r}(Q) & =-\nabla U_{i}^{r}(Q) \\
& =\left\{\begin{array}{l}
k_{i}^{r} \frac{\left(Q-Q_{i}^{o}\right)}{d_{i}(Q)^{3}}\left(\frac{1}{d_{i}(Q)}-\frac{1}{d_{i}^{o}}\right) \text { if } d(Q) \leq d_{i}^{o} \\
0 \quad \text { otherwise }
\end{array}\right.
\end{aligned}
$$

Sometimes, the robot's final position is too close to an obstacle. The robot environment configuration creates local minima in the neighborhood of the global state and makes this global node not reachable. The solution to such issues, is to multiply the repulsive potential, according to each obstacle, by the distance between the current state and the goal.

$U_{i}^{r}(Q)=\left\{\begin{array}{l}\frac{1}{2} k_{i}^{r} d_{i}(Q)\left(\frac{1}{d_{i}(Q)}+\frac{1}{d_{i}^{o}}\right)^{2} \text { if } d(Q) \leq d_{i}^{o} \\ 0 \quad \text { otherwise }\end{array}\right.$

The total potential field and forces are the sum of the two attractive and repulsive fields and forces respectively.

$\left\{\begin{array}{l}U^{t}(Q)=U^{a}(Q)+U^{r}(Q)=U^{a}(Q)+\sum_{i=1}^{n} U_{i}^{r}(Q) \\ f^{t}(Q)=f^{a}(Q)+f^{r}(Q)=f^{a}(Q)+\sum_{i=1}^{n} f_{i}^{r}(Q)\end{array}\right.$

\section{Potential Field Method ANd Local Minima}

The potential field method is still sensitive to narrow paths, which induce chattering issues, and to local minima, which are a source of blocking configurations [8-10]. The major drawback in APF method is the possibility of getting trapped by these local minima [12]. They are commonly related to the robot's workspace configuration and especially to weight coefficients, associated to each obstacle during the APF design [11], when attractive forces cancel the repulsive ones. Numerous approaches are put 
forward to overcome the local minima issue, thus, it remains a difficult problem. Barraquand et al. proposed in [21] to move the robot randomly at each time it gets trapped in a local minimum. Therefore, the determined path usually loses some of its smoothness and some configurations take a long time to get the robot far away from the local minimum. Some other solutions suggested driving the robot along the nearest barriers in the same clockwise direction each time the robot blocked by a local minimum. P. Vadakkepat et al [10] proposed an evolutionary artificial potential field combined with global optimization algorithms, genetic algorithms, to adjust the APF online. However, the first suggestion is only available for narrow spaces and minor local minima. The second is insufficient for the robot planning trajectory considering the robot dynamic. And the third is often time-consuming. Some others propose adding attraction points in some of the vertices of the obstacles to get the robot out of local minima. According to the same principle, the objective of the new approach, which is presented in this paper, is to overcome and eliminate the local minima. The idea is inspired from pouring a liquid matter, water for instance, with high pressure - attractive artificial potential fieldfrom the initial state until it reaches the global state. The proposed strategy consists of designing the total APF as the classic method according to the two sets of equations (1) and (7). The strategy uses the potential gradient descent algorithm (PGDA) to discover the robot path. The PGDA is executed as long as the ongoing state is different from the global minimum. If it cannot go forward, trapped in a local minimum, then the algorithm adds some extra repulsive potential to the current state until it gets free, according to the set of equations (7). Once the robot's destination is reached, the PGDA searches, once again, the final and the practicable robot path in the new potentialfield. The advantage of this proposed method is that the attractive and the repulsive APF are computed just once according to the final and to the initial robot position,

\section{The standard artificial potential field shape}

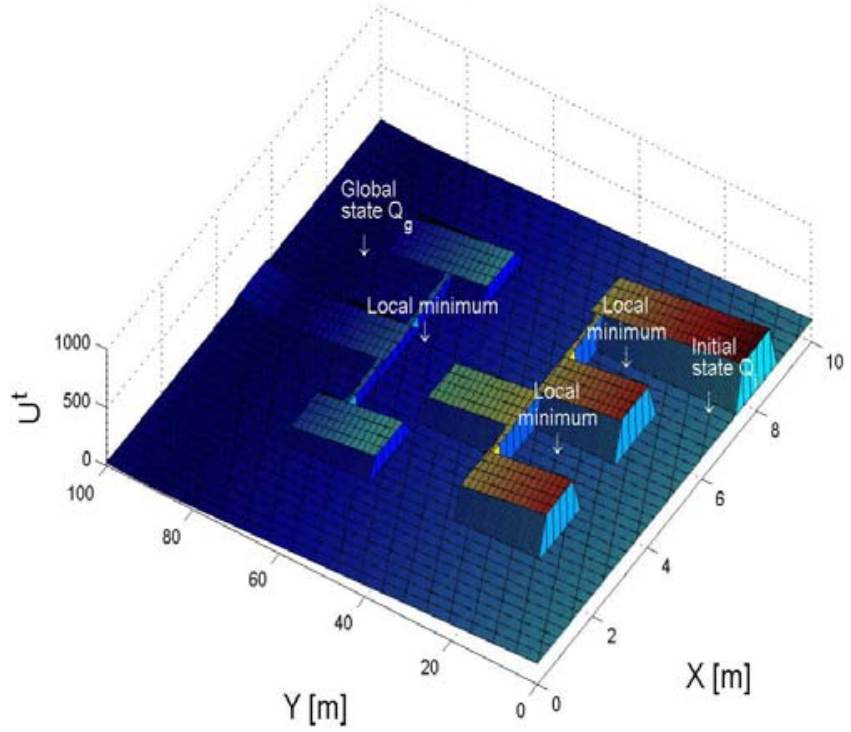

Figure 1: Standard potential field shape. respectively, rather than computing the repulsive APF, associated to each obstacle, at each current position of the robot when searching the path, according to the equation (4). These modifications make the APF method faster but they do induce some limitations. For instance, if the robot's workspace configuration creates a path with sharp corners, the method may partially and even completely drown the path and the robot will definitely be trapped.

The proposed potential field method skeleton for robot trajectory planning is described as follows:

1. Design the attractive $P F$ " $U$ " " according to global state.

2. Design the set of the repulsive $P F$ " $U_{i}^{r}$ " according to each obstacle with its parameters: " $k_{i}^{r}$ " and " $d_{i}{ }^{\text {" ". }}$

3. Assign the initial state $Q_{i}$ to the path vector.

4. while $(d(Q) \neq 0)$

4.1. Call the potential gradient descent algorithm (PGDA) to determine the next state.

4.2. Add the current state to the path vector.

4.3. if (robot is blocked $\& \& d(Q) \neq 0$ ) //local minima Add some additional new repulsive $P F$ " $U_{i}^{r}$ " to the total PF " $U^{t}$ " according to the local end if minimum parameters " $k_{i}^{o}$ min" and " $d_{i}^{o}{ }_{\min }$ ".

end while

5. Call the potential gradient descent algorithm (PGDA) to determine the new path.

where: " $k_{i}^{o}$ min " and " $d_{i}^{o}$ min" are the distance of influence and the gravitational constant of the local minimum.

\section{Simulation Strategy and PRELIMINARy RESUlts}

To test the proposed method, the robot workspace of ten meters wide and one hundred meters long is created with MATLAB R2012 on windows 8.1 and Intel compute Intel ${ }^{\circledR}$ Core $^{\mathrm{TM}}$ i5-3320M, CPU @ $2.60 \mathrm{GHz}$ and RAM 16Go-. Multiple obstacles produce three different local minima with different sizes between the initial robot position and its distinction, as illustrated in figures 1-4.

\section{The modified artificial potential field shape}

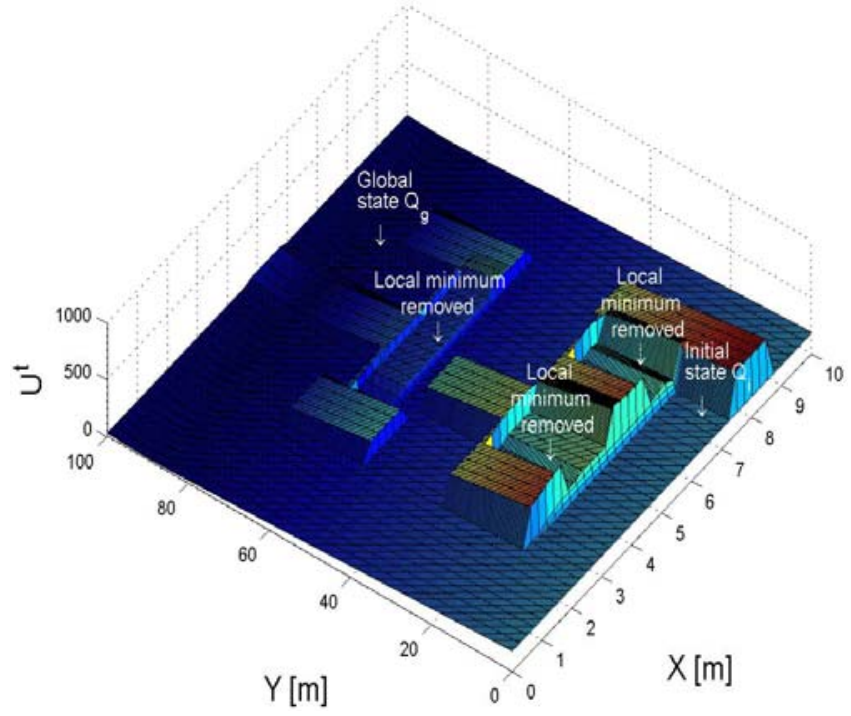

Figure 2: Modified potential field shape. 


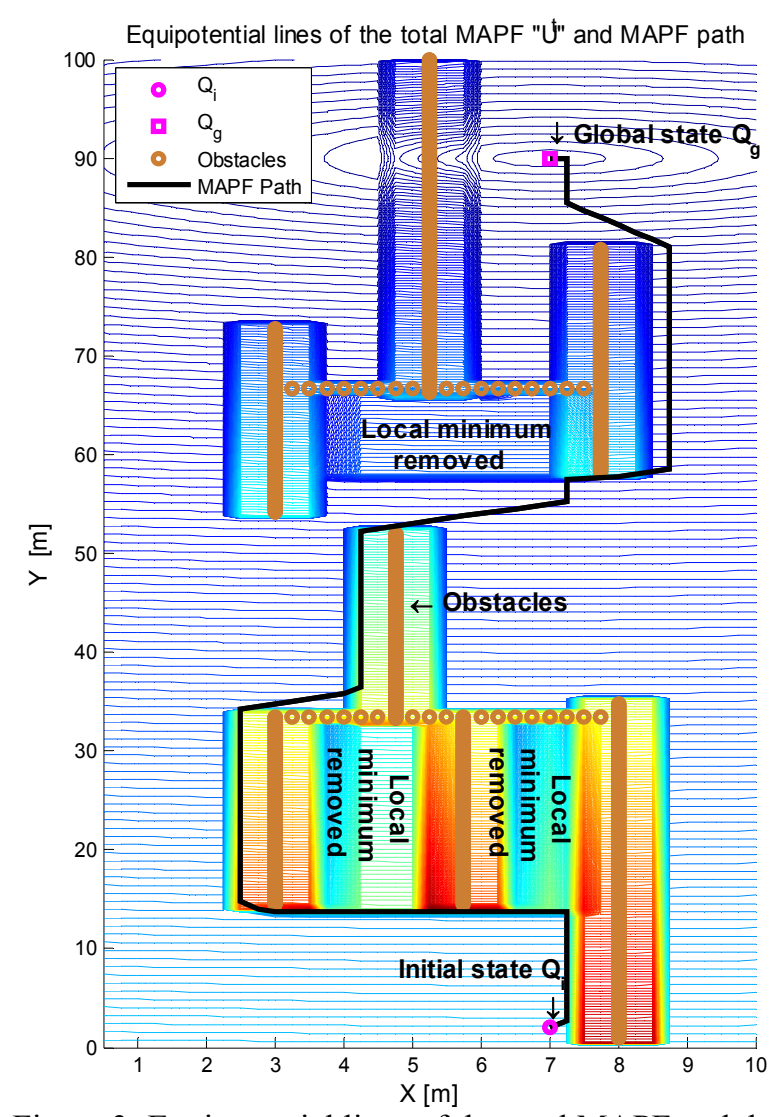

Figure 3: Equipotential lines of the total MAPF and the MAPF path.

The area is divided into squares of 0.25 by $0.25 \mathrm{~m}$. As previously noted, in case of the use of the standard APF method, the potential gradient descent algorithm is attracted and blocked by the nearest minimum even after adding random noise, this is due to the local minima, figure 1 . So to avoid the global optimization and following the obstacles methods, the modified APF approach gradually eliminates the local minima as shown in figure 2 during the first execution of the PGDA. Each time the robot gets blocked, additional repulsive APF are added to the current state and to its neighbors according to its specified distance of influence $d_{i}^{o}$ min and its gravitational coefficient $k_{i}^{o}$ min. It is possible to see the position of each local minimum as depicted in figure 1, and the drowned ones in figures 2 and 3. The second operation of PGDA determines the satisfactory path that will be a reference path for robot trajectory. The equipotential lines of the total MAPF, in figure 3, show how the suggested method changes the equipotential lines in the local minima by changing the value of the repulsive APF at each time the robot is blocked. Figure 4 shows the three different paths determined by the proposed methods, at the first run of the MAPF method with local minima and after drowning them, and finally the determined path by the standard $\mathrm{A}^{*}$ method. The first run of the MAPF goes through all local minima and drowns them gradually as the robot gets closer to its target position, represented by small blue circles. Once there, the second execution of the PGDA selects the best path according to the new APF drawn by the black line,

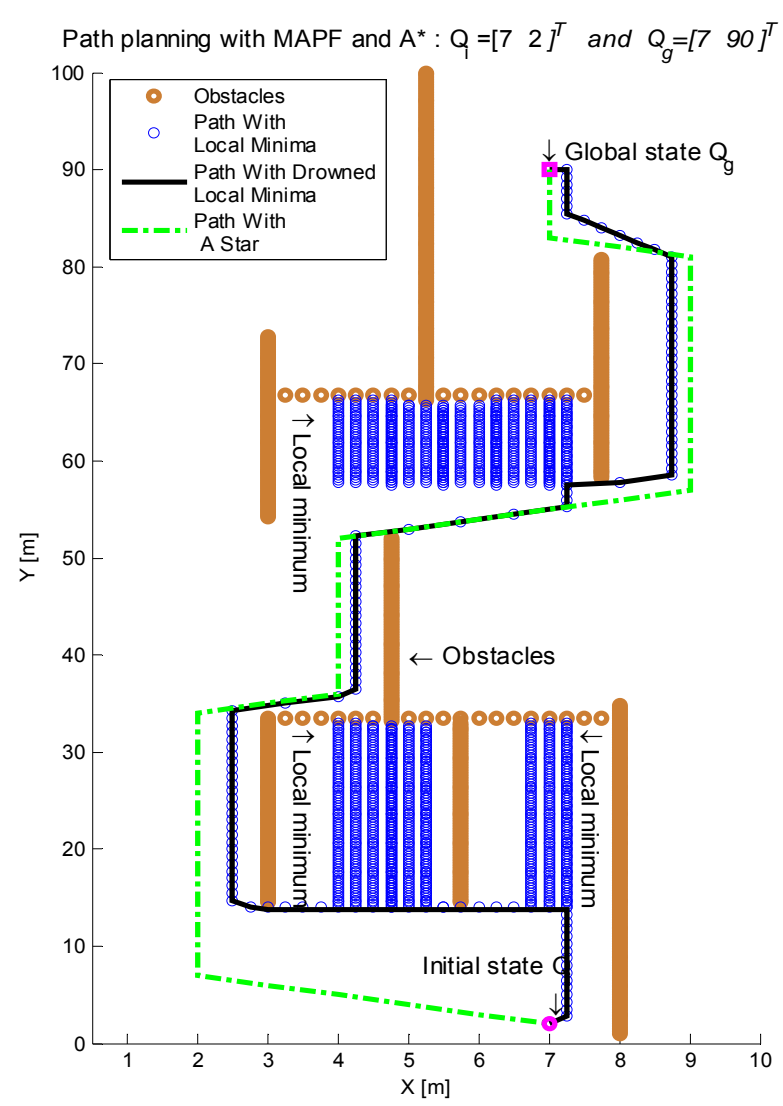

Figure 4: Path planning with MFP and A*.

and, finally, the $\mathrm{A}^{*}$ path is represented by the green dashed line. The respective mean execution time of 100 runs of the proposed modified artificial potential field and of the standard $A^{*}$ methods, using the same robot workspace, are 282.34 and $218.59 \mathrm{~ms}$, that means $\mathrm{A}^{*}$ is still faster than MAPF with a difference of $63.75 \mathrm{~ms}$ for this simulation scenario. The path searching of each method depends on the robot's workspace size and its configuration, the size of the barriers, obstacles, and the number and the size of the local minima between the robot and its final destination for the MAPF method. Both methods (MAPF and A*) could be improved to take in account the robots geometries by changing the value of the distance of influence of each obstacle according to the size of the robot, in the case of the APF method, and changing the size of the obstacle for $\mathrm{A}^{*}$ and its variants as proposed for instance in [16].

\section{CONCLUSION}

This paper presented a modified artificial potential field method toward online path planning. The approach adds dynamically repulsive artificial potential field, to the standard APF, at each the robot gets trapped in a local minimum. The operation eliminates any blocking configuration, in the robot environment, that is the major challenge of the standard APF method. This operation is continuously repeated as long as the final destination of the robot is not reached. Finally, the strategy uses the potential gradient descent algorithm to determine the new feasible path according to the first execution. The simulation 
results, the comparison between the $A^{*}$ and MAPF performances, show the effectiveness of the suggested strategy and its alignment with real-time path planning. However, it is worthy to emphasis on the fact that the suggested MAPF method computation time depends on the number and the size of the local minima that PGDA meets at the first run. A solution for such as problems is to associate an appropriate distance of influence $d_{i}{ }^{o}$ min of each local minimum, according to its size. Another drawback of the method is sharp corners and the position of the robot and its target that may induce the MAPF method to drown partially and even completely the sharp S-shaped trajectories during the first execution. The future work will focus on the implementation of the modified potential field method for vehicle local navigation.

Table 1: Potential field variables

\begin{tabular}{|c|c|c|c|}
\hline Variables & Value & Variables & Value \\
\hline$d_{i}{ }^{o}$ & $0.5[\mathrm{~m}]$ & $k^{a}, k_{i}{ }^{o}$ & 1 \\
\hline$d^{r}{ }_{i, \min }$ & $0.5[\mathrm{~m}]$ & $k_{i, \min }^{r}$ & 0.05 \\
\hline
\end{tabular}

\section{ACKNOWLEDGMENT}

This work is part of COOPERCOM, a 3-year international research project (Canada-France). The authors would like to thank the firms ESI-Civitec in France and Opal-RT in Canada, the MITACs Accelerate Canadian funding program, the National Science and Engineering Research Council (NSERC) of Canada and the Agence nationale de la recherche (ANR) in France for supporting the project.

\section{REFERENCES}

[1] Steaven M. LaValle, "Planning Algorithms.", Cambridge University Press.”, 2006

[2] Jong-Hwan Kim, Yong-Duk Kim, and Kang-Hee Lee "The Third Generation of Robotics: Ubiquitous Robot." 2nd International Conference on Autonomous Robots and Agents, Palmerston North, New Zealand, Dec 13-15, 2004.

[3] Miguel A. Padilla Castaneda, Jesus Savage, Adalberto Hernandez and Fernando Arambula Cosío (2008). "Local Autonomous Robot Navigation Using Potential Fields, Motion Planning.", Xing-Jian Jing (Ed.), ISBN: 978-9537619-01-5, In Tech

[4] You Li, Yassine Ruichek and Cindy Cappelle," 3D Triangulation Based Extrinsic Calibration between a Stereo Vision System and a LIDAR." $14^{\text {th }}$ International IEEE Conference on Intelligent Transportation Systems Washington DC, USA. Oct 5-7, 2011.

[5] P. Nunez, P. Drews Jr, R. Rocha, and J. Dias. "Data fusion calibration for a $3 d$ laser range finder and a camera using inertial data" in Proc. of 4th European Conf. on Mobile Robots (ECMR2009), Sep.2009, pp. 31-36.

[6] H. Aliakbarpour, P. Nez, J. Prado, K. Khoshhal, and J. Dias, "An efficient algorithm for extrinsic calibration between a $3 d$ laser range finder and a stereo camera for surveillance." in 14th International Conference on Advanced Robotics (ICAR 2009), 2009.
[7] Z. Zhang, "A flexible new technique for camera calibration" IEEE Transactions on Pattern Analysis and Machine Intelligence, vol. 22, pp. 1330-1334, 2000.

[8] D. Yagnik, J. Ren, and R. Liscano, "Motion planning for multi-link robots using artificial potential fields and modified simulated annealing." ASME International Conference on Mechatronics and Embedded Systems and Applications, July 2010.

[9] E. Rimon and D. Koditschek, "Exact robot navigation using artificial potential functions." IEEE Transactions on robotics and automation, vol. 8, 1992.

[10] P. Vadakkepat; Kay Chen Tan; Wang Ming-Liang: "Evolutionary artificial potential fields and their application in real time robot path planning" Proceedings of the 2000 Congress on Evolutionary Computation, 16-19 July 2000.

[11] O. Khatib. "Real-time obstacle avoidance for manipulators and mobile robots." The international Journal of Robotic Research, Vol. 5, No 1, Spring 1986.

[12] Hao-Tien Chiang, Nick Malone, Kendra Lesser, Meeko Oishi, Lydia Tapia: "Path-Guided Artificial Potential Fields with Stochastic Reachable Sets for Motion Planning in Highly Dynamic Environments." International Conference on Robotics and Automation (ICRA), Washington. May 26-30, 2015.

[13] Ferry Rippun Gideon Manalu: "Double Target Potential Field Planning the Movement on Passing and Kicking the Ball in Soccer Robot" sixth International Conference on Information Technology and Electrical Engineering (ICITEE), 7-8 Oct. 2014, Yogyakarta, Indonesia.

[14] Jianhao Tan, Luodi Zhao, Yaonan Wang, Yiwei Zhang, Lu Li: "The 3D Path Planning Based on A* Algorithm and Artificial Potential Field for the Rotary-Wing Flying Robot." Eighth International Conference on Intelligent HumanMachine Systems and Cybernetics, 27-28 Aug. 2016.

[15] Ge, S.S., Cui, Y.J.: "New potential functions for mobile robot path planning." IEEE Trans. Robot. Automat. NO. 5, October. 2000

[16] Dolgov, D., Thrun, S., Montemerlo, M., Diebel, J.: "Path planning for autonomous vehicles in unknown semistructured environments." Int. J. Robot. Res. 29(5) (2010)

[17] Qiang Song, Lingxia Liu: "Mobile Robot Path Planning Based on Dynamic Fuzzy Artificial Potential Field Method." International Journal of Hybrid Information Technology Vol. 5, No. Oct. $4^{\text {th }} 2012$.

[18] Xiang Liu, "A comparative study A-Star algorithms for search and rescue in perfect maze." International Conference on Electric Information and Control Engineering (ICEICE), Wuhan, China, 15-17 April 2011.

[19] Yizhen Huang, Qingming Yi, Min Shi, "The improved Dijkstra's shortest path algorithm.", 2011 Seventh International Conference on Natural Computation (ICNC), Shanghai, China, 26-28 July 2011

[20] Takayuki Goto, Takeshi Kosaka, and Hiroshi Noborio, "On the Heuristics of A-Star or A Algorithm in ITS and Robot Path-Planning." Proceedings of the 2003, RSJ International Conference on Intelligent Robots and Systems, Oct 2003.

[21] Barraquand, J., Latombe, J.C. "Robot motion planning: A distributed representation approach." The International Journal of Robotics Research, December 1991. 